\title{
Sol-Gel Transition during Inclusion Complex Formation between $\alpha$-Cyclodextrin and High Molecular Weight Poly(ethylene glycol)s in Aqueous Solution
}

\author{
Jun LI, Akira HaradA*, and Mikiharu Kamachi* \\ Department of Macromolecular Science, Faculty of Science, \\ Osaka University, Toyonaka, Osaka 560, Japan
}

(Received January 5, 1994)

\begin{abstract}
Poly(ethylene glycol)s (PEG) of high molecular weights were found to form complexes with $\alpha$-cyclodextrin $(\alpha-C D)$ in aqueous solutions to give gels in a wide range of concentrations. The time of gelation decreases with increase in $\alpha-\mathrm{CD}$ and PEG concentrations, indicating that the gels formed during complex formation between $\alpha-\mathrm{CD}$ and PEG chains. The time of gelation increases with increase in the molecular weight of PEG, indicating that the PEG chains penetrate $\alpha$-CD cavities from the ends of PEG and are included by $\alpha$-CDs. X-Ray powder diffraction studies showed that the gel consists of both complexed $\alpha-C D$ and uncomplexed $\alpha-C D$, indicating partial inclusion of PEG chains by $\alpha-\mathrm{CD}$. The configuration of the gels and mechanism for the gelation are discussed, and it is suggested that $\alpha$-CD-PEG complexes act as physical cross-links during gelation. The gel-melting temperature increases with increase in PEG molecular weights and $\alpha-C D$ concentrations, and decreases with increase in PEG concentrations, suggesting that gelation results from the formation of longer or shorter domains of $\alpha$-CD-PEG inclusion complexes, respectively.
\end{abstract}

KEY WORDS Sol-Gel Transition / $\alpha$-Cyclodextrin / High Molecular Weight Poly(ethylene glycol) / Inclusion Complex / X-Ray Diffraction Pattern /

Cyclodextrins $(\alpha-\mathrm{CD}, \beta-\mathrm{CD}$, and $\gamma$-CD) (Figure 1a) are cyclic oligosaccharides with internal cavities capable of forming inclusion complexes with small organic or inorganic compounds in aqueous solutions and a great number of inclusion complexes have been prepared and characterized. ${ }^{1,2}$ They have been studied extensively as models for enzyme active sites with the intention of mimicking enzyme activity and understanding the mechanism of molecular recognition. ${ }^{1-4}$ However studies in these fields were limited to low molecular weight compounds, and there were no reports on the complex formation of $\mathrm{CDs}$ with polymers until we focused our research on interactions of CDs with polymers and found that $\alpha-C D$ selectively forms inclusion complexes with poly(ethylene glycol)s (PEG). ${ }^{5}$

As described in previous papers ${ }^{5}$, when a saturated aqueous solution of $\alpha$-CD was added to aqueous solutions of PEG at room temperature, the solutions became turbid and

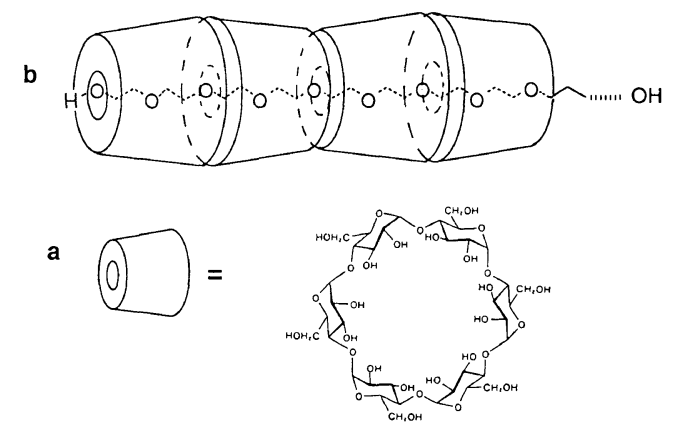

Figure 1. (a) Bucket-like structure of $\alpha-C D$ with hydrophobic cavity and hydrophilic face. There are 6 primary hydroxyl groups on the narrow side and 12 secondary hydroxyl groups on the wide side. (b) Channel structure of $\alpha$-CD-PEG inclusion complex with many $\alpha$-CD threaded on a PEG chain. In each $\alpha-\mathrm{CD}$ cavity are bound two ethylene glycol units. 
the crystalline $\alpha$-CD-PEG inclusion complexes formed. When the average molecular weights of PEG are less than 2000, we obtained stoichiometric complexes in which two ethylene glycol units of PEG are bound in a single $\alpha-C D$ cavity. This stoichiometry was confirmed by ${ }^{1} \mathrm{H}$ NMR spectra of the complexes and continuous variation method for complex formation as well as studies on the size of the guest relative to that of the $\alpha-C D$ cavity. The X-ray powder patterns of $\alpha$-CD-PEG complexes showed that the complexes were isomorphous with those of channel-type structure, and it is suggested that PEG chains penetrate $\alpha$-CD cavities; then the $\alpha-C D$ rings connect together by the hydrogen bonds between one another to form the stoichiometric $\alpha$-CD-PEG inclusion complexes. The $\alpha-\mathrm{CD}$ rings become hydrophobic because of the consumption of the hydroxyl groups, and interactions of $\alpha$-CD-PEG complexes result in crystallization. Therefore, it is concluded that $\alpha$-CD-PEG complexes take structures like Figure $1 \mathrm{~b}$ with many $\alpha-C D$ rings threaded stoichiometrically by a PEG chain.

We found here that sol-gel transition takes place during formation of inclusion complexes between $\alpha-C D$ and PEG in aqueous solutions, when the molecular weights of PEG are higher than 2000. This paper discusses the sol-gel transition conditions, time of gelation, and gel-melting temperature. We also carried out $\mathrm{X}$-ray diffraction measurements on the gels. The most probable mechanism for the gelation of $\alpha-C D$ and PEG aqueous solutions is discussed.

\section{EXPERIMENTAL}

\section{Materials}

$\alpha$-Cyclodextrin was purchased from Nakarai Tesque Co., Ltd. and dried under vacuum. Poly(ethylene glycol)s (PEG) with various average molecular weights were used. $\mathrm{Ab}$ breviation, average molecular weight, $\overline{M W}$, average number of ethylene glycol units, $\overline{D P}$, and manufacturer of the different PEG samples are listed in Table I. Average molecular weights of the PEG samples were found by GPC (gel permeation chromatography) to be within specifications of the suppliers.

\section{Gel Formation}

$\alpha$-CD-PEG gels were prepared by mixing $\alpha-C D$ aqueous solution and PEG aqueous solutions with various average molecular weights followed by sonication and standing at $20^{\circ} \mathrm{C}$ for at least 90 hours. Sol-gel transition occurred while $\alpha-C D$ forms inclusion complexes with PEG chains if suitable concentrations of $\alpha-C D$ and PEG are used.

\section{Gel-Melting Temperature Measurements}

The gel-melting temperature was measured by the tilting method. ${ }^{6}$ The gels were subjected to slow programmed temperature increase $(0.5$ $\left.{ }^{\circ} \mathrm{C} \min ^{-1}\right)$ in test tubes. The temperature of initiation of the gels flowing under their own

Table I. PEG samples and average molecular weights, $\overline{M W}$.

\begin{tabular}{|c|c|c|c|}
\hline Abbreviation & $\overline{M W}$ & $\overline{D P}$ & Manufacturer \\
\hline PEG $\# 2000$ & 2000 & 40 & Naclai Tesque Co., Ltd. \\
\hline PEG $\# 3350$ & 3350 & 50 & Sigma Chemical Co. \\
\hline PEG $\# 8500$ & 8000 & 190 & Tokyo Kasei Kogyo Co., Ltd. \\
\hline$P E G \sharp 20000$ & 20000 & 450 & Wako Pure Chemical Ind., Ltd. \\
\hline PEG $\# 50000$ & 50000 & 1140 & Wako Pure Chemical Ind., Ltd. \\
\hline PEG $\# 100000$ & 100000 & 2270 & Aldrich Chemical Co., Inc. \\
\hline PEG $\# 300000$ & 300000 & 6820 & Aldrich Chemical Co.. Inc. \\
\hline PEG $\# 600000$ & 600000 & 13640 & Aldrich Chemical Co., Inc. \\
\hline PEG $\# 900000$ & 900000 & 20450 & Aldrich Chemical Co., Inc. \\
\hline
\end{tabular}


weight in the tubes when the upright test tubes were tilted was taken as the gel-melting temperature. The gels became mobile suspensions above the gel-melting temperature.

\section{$X$-Ray Diffraction Measurements}

Gels with various PEG molecular weights were formed from the $\alpha-C D-P E G$ aqueous solutions containing $11.0 \mathrm{gl}^{-1}$ of PEG and $120.8 \mathrm{~g} \mathrm{l}^{-1}$ of $\alpha-\mathrm{CD}$ in which the molar ratio of ethylene glycol units to $\alpha-\mathrm{CD}$ was $2: 1$. The gels were freeze-dried and ground into fine powder. A frozen aqueous solution of 120.8 $\mathrm{g} 1^{-1} \alpha$-CD was freeze-dried and ground into fine powder. X-Ray diffraction patterns of the powders were taken using a Rigaku RDAROC X-ray diffractometer. The operation conditions were as follows: $\mathrm{X}$-ray, nickelfiltered $\mathrm{Cu}-K_{\alpha}$ radiation $(\lambda=1.542 \AA)$; voltage, $40 \mathrm{kV}$; current, $40 \mathrm{~mA}$; scanning speed, $3^{\circ}$ per min.

\section{RESULTS}

\section{Sol-Gel Transition Curve}

Mixed aqueous solutions of PEG and $\alpha-C D$ have been found to form gels when left at room temperature for long enough, and when the molecular weights of PEG are larger than 2000 and concentrations of $\alpha-C D$ and PEG are high enough. The time of gelation and minimum concentrations of $\alpha-\mathrm{CD}$ and PEG required for gelation varies with the molecular weight of PEG. As a typical example, the sol-gel transition phase diagram for the aqueous solution of PEG $\# 20000$ and $\alpha$-CD is shown in Figure 2. The open circle in Figure 2 means that the solution formed gel and the cross means that the solution was still a sol after standing 90 hours or longer. On standing 90 hours, the sol-gel transition curve was almost identical with that shown in Figure 2. This means that a $\alpha$-CD-PEG aqueous solution forms a gel when the concentrations of $\alpha$-CD and PEG are in the right and above area of the sol-gel transition curve.

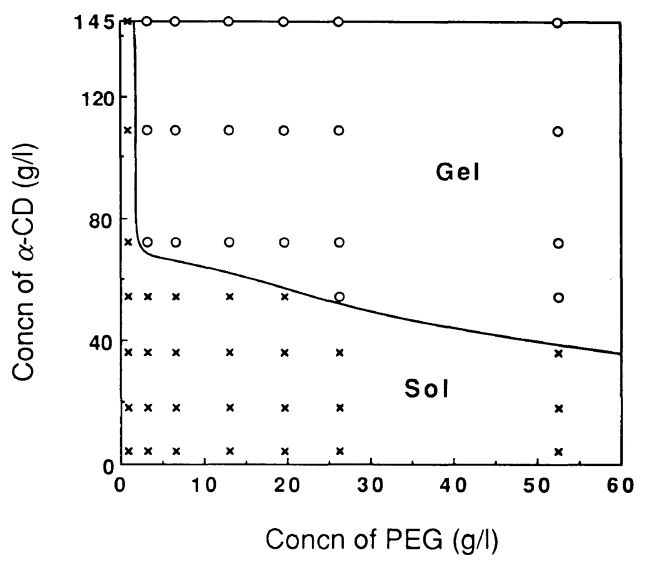

Figure 2. Phase diagram for the sol-gel transition of aqueous $\alpha$-CD-PEG solution. The molecular weight of PEG is 20000 . The open circle " $O$ " means that the solution formed gel within 90 hours, and the cross " $x$ " means that the solution did not form gel after standing 90 hours or longer at $20^{\circ} \mathrm{C}$. The sol-gel transition curve is drawn between the sol area and gel area.

In Figure 2, as the concentration of PEG decreases, a higher concentration of $\alpha-C D$ is required for gelation. When the concentration of PEG is less than $2.0 \mathrm{gl}^{-1}$, gelation does not occur even though $\alpha-\mathrm{CD}$ is saturated, i.e., the concentration of $\alpha$-CD is $145 \mathrm{~g}^{-1}$. $\alpha$-CD-PEG gels could become fluids by heating over gel-melting temperature or agitating them and gelation occurred again when they were allowed to stand below the gel-melting temperature. $\alpha-C D-P E G$ gels are thermoreversible gels.

\section{Time for the Gelation}

Figure 3 shows the effect of PEG concentration on the gelation time of aqueous $\alpha-C D$ PEG $\$ 20000$ solutions. As the concentration of PEG increased, the time of gelation decreased.

Figure 4 shows the effects of the concentration of $\alpha-C D$ on the time of gelation of aqueous $\alpha$-CD-PEG $\# 20000$ solutions containing 6.56 $\mathrm{g}^{-1}$ of PEG $\# 20000$. The time of gelation decreased as the concentration of $\alpha-C D$ increased. Figures 3 and 4 show that the gels formed in a few minutes when the concentrations of $\alpha-C D$ or PEG were large enough.

Figure 5 shows the time required for gelation 


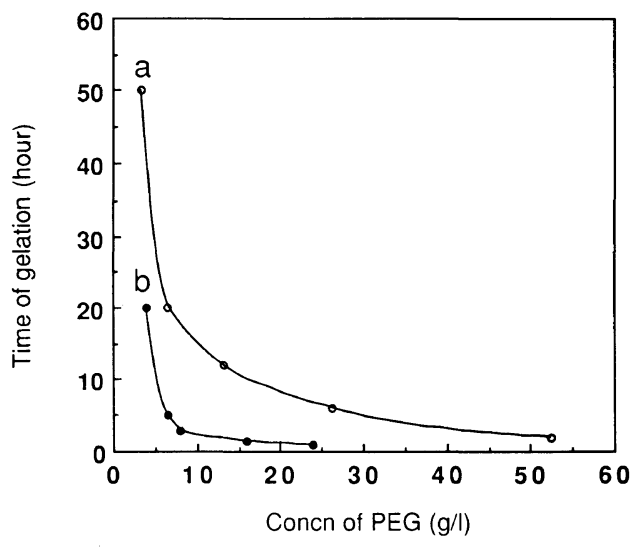

Figure 3. Time for the gelation of $\alpha$-CD-PEG $\# 20000$ aqueous solution as a function of PEG concentration. The concentrations of $\alpha-\mathrm{CD}$ are $72.5 \mathrm{gl}^{-1}$ (a) and $87.0 \mathrm{gl}^{-1}$ (b). The gel formation temperature is $20^{\circ} \mathrm{C}$.

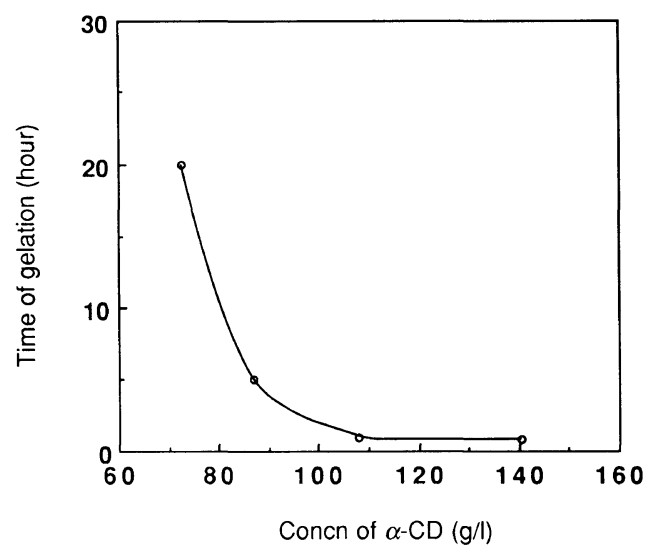

Figure 4. Time for the gelation of $\alpha$-CD-PEG $\# 20000$ aqueous solution as a function of the concentration of $\alpha$-CD. The concentration of PEG is $6.56 \mathrm{gl}^{-1}$ and gel formation temperature, $20^{\circ} \mathrm{C}$.

as a function of PEG molecular weight from 2000 to 50000 . The gel contained $72.5 \mathrm{~g}^{-1}$ of $\alpha-\mathrm{CD}$ and $6.56 \mathrm{~g} \mathrm{l}^{-1}$ of PEG. It should be noted that the time of gelation increased with increase in the PEG molecular weight.

\section{$X$-Ray Diffraction Powder Patterns}

Aqueous solutions of $\alpha-C D$ and PEG of molecular weight higher than 2000 formed gels rather than crystalline complexes in a solid state that could be isolated from the aqueous so-

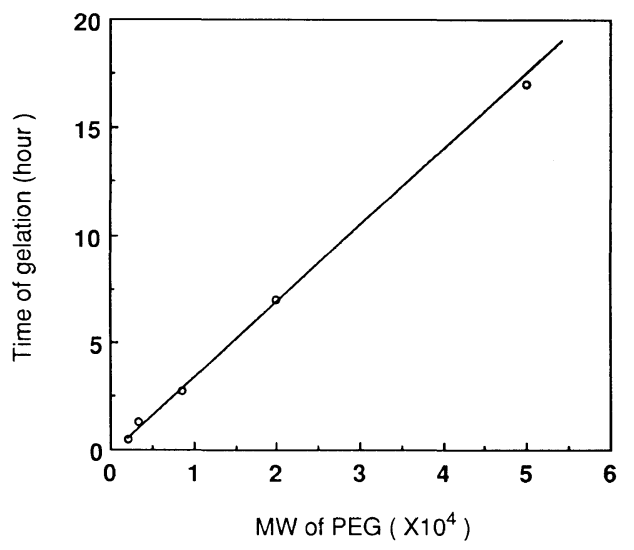

Figure 5. Time for the gelation of $\alpha-C D-P E G$ aqueous solution as a function of the molecular weight of PEG. The solutions contain $72.5 \mathrm{gl}^{-1}$ of $\alpha-\mathrm{CD}$ and $6.56 \mathrm{gl}^{-1}$ of PEG. The gel formation temperature is $20^{\circ} \mathrm{C}$.

lutions. In order to investigate the structures of the gels, we measured the X-ray diffraction powder patterns of the freeze-dried gels with various PEG molecular weights from 2000 to 4000000. The patterns are shown in Figure $6 \mathrm{a}-\mathrm{h}$. All the gels in Figure 6 were formed from aqueous solutions containing $11.0 \mathrm{gl}^{-1}$ of PEG and $120.8 \mathrm{gl}^{-1}$ of $\alpha-\mathrm{CD}$ in which the molar ratio between ethylene glycol units and $\alpha$-CD was $2: 1$. For comparison, the $\mathrm{X}$-ray diffraction diagram of the freeze-dried aqueous solution of $\alpha-\mathrm{CD}\left(120.8 \mathrm{gl}^{-1}\right)$ is shown in Figure 6i. Two very weak and broad peaks of the freeze-dried samples suggest that the frozen solution of $\alpha-C D$ has a random alignment structure. As described in previous papers, ${ }^{5}$ in contrast, the X-ray diffraction pattern showing a number of sharp reflection lines and one strong and sharp main reflection line for $2 \theta=19.9^{\circ}$ is identical with the extended column structure or channel structure of $\alpha-C D$ which corresponds to the crystalline $\alpha$-CD-PEG inclusion complexes in cases for PEG molecular weights of less than 2000. The diffraction pattern of $\alpha$-CD-PEG $\# 2000$ gel shown in Figure $6 \mathrm{a}$ is similar to the so-called channel structure pattern. The reflection line for $2 \theta=19.9^{\circ}$ appeared in all of the diffraction 


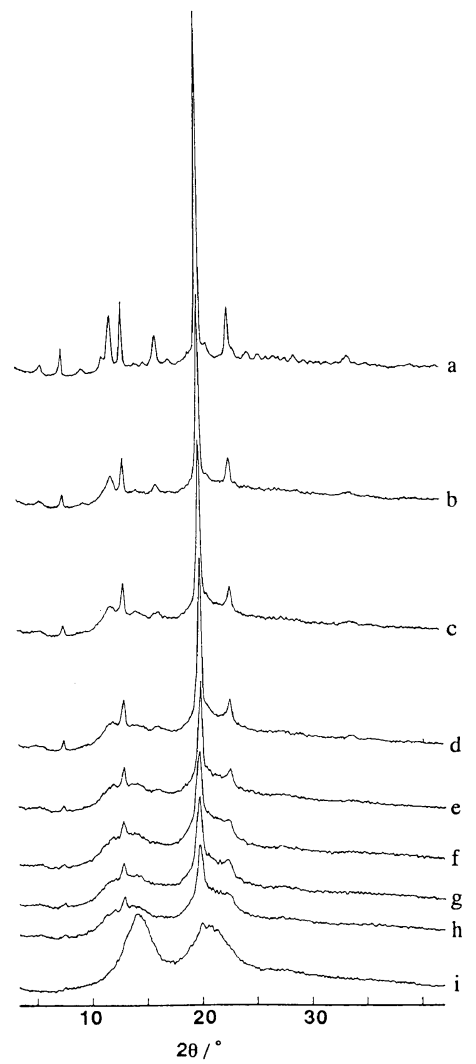

Figure 6. X-ray diffraction powder patterns for freezedried $\alpha$-CD-PEG $\$ 2000$ gel (a), $\alpha$-CD-PEG $\$ 8500$ gel (b), $\alpha$-CD-PEG $\# 20000$ gel (c), $\alpha$-CD-PEG $\$ 50000$ gel (d), $\alpha$-CD-PEG $\# 100000$ gel (e), $\alpha$-CD-PEG $\# 300000$ gel (f), $\alpha$-CD-PEG $\$ 600000$ gel (g), $\alpha$-CD-PEG $\$ 900000$ gel (h), and freeze-dried aqueous $\alpha$-CD solution (i). The gels are formed from aqueous solutions containing $11.0 \mathrm{gl}^{-1}$ of PEG and $120.8 \mathrm{gl}^{-1}$ of $\alpha-\mathrm{CD}$ in which the molar ratio of ethylene glycol units to $\alpha-C D$ is $2: 1$.

diagrams of the $\alpha$-CD-PEG gels, and showed a decrease in strength with increase in the PEG molecular weight. The pattern of random structure of $\alpha-C D$ with two weak and broad peaks appeared in these same diffraction diagrams, and showed increase in strength with increase in PEG molecular weight. It is obvious that the gel system consists of two kinds of $\alpha-\mathrm{CD}$, the channel structure $\alpha-\mathrm{CD}$ and random structure $\alpha-C D$, which indicates that there are both complexed $\alpha-C D$ and uncomplexed $\alpha-C D$ in the gel system, and that higher PEG

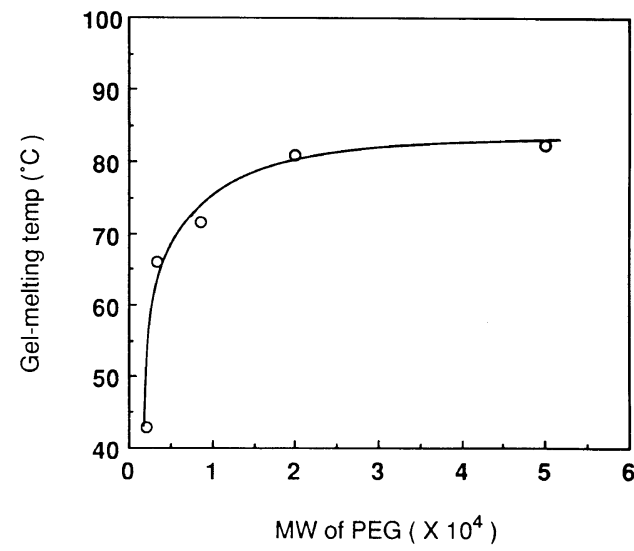

Figure 7. Gel-melting temperature as a function of molecular weight of PEG. The gels are formed at $20^{\circ} \mathrm{C}$ from aqueous solutions containing $6.56 \mathrm{gl}^{-1}$ of PEG and $72.5 \mathrm{gl}^{-1}$ of $\alpha$-CD.

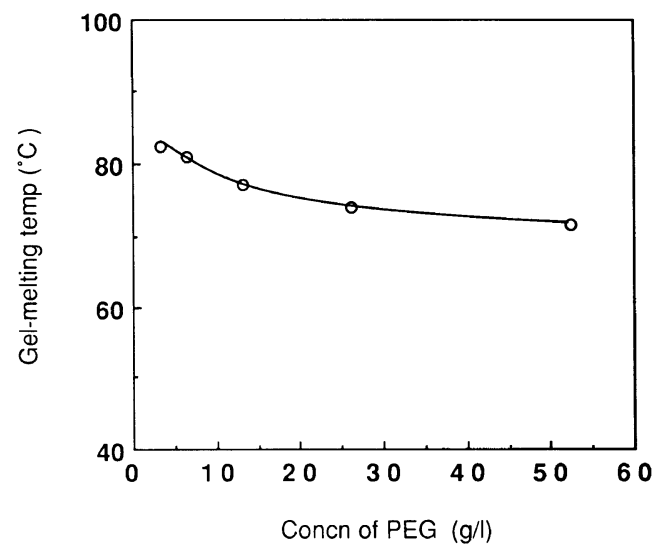

Figure 8. Gel-melting temperature as a function of concentration of PEG. The gels formed at $20^{\circ} \mathrm{C}$ and contain $72.5 \mathrm{~g}^{-1}$ of $\alpha-\mathrm{CD}$. The molecular weight of PEG is 20000.

molecular weight causes a decrease in the ratio of complexed $\alpha-\mathrm{CD}$ to uncomplexed $\alpha-\mathrm{CD}$.

\section{Gel-Melting Temperature}

Figure 7 shows how gel-melting temperature is effected by the molecular weight of PEG. The plots clearly show that the gel-melting temperature increased with increase in the molecular weight of PEG. Particularly, the gelmelting temperature showed a sharp increase in the molecular weight of PEG from 2000 to 20000 . 
The gel-melting temperature for the gels was also effected by the concentration of $\alpha$-CD. For example, for $\alpha$-CD-PEG $\$ 20000$ gels formed from $6.56 \mathrm{~g}^{-1}$ of PEG and various concentrations of $\alpha-\mathrm{CD}$, the gel-melting temperature was $81.5^{\circ} \mathrm{C}$ when the concentration of $\alpha-\mathrm{CD}$ was $72.5 \mathrm{gl}^{-1}$; and $89.1^{\circ} \mathrm{C}$ when the concentration of $\alpha$-CD was $140.5 \mathrm{gl}^{-1}$. The gel-melting temperature gradually increased with increase in the $\alpha-\mathrm{CD}$ concentration.

The effect of the PEG concentration on the gel-melting temperature for the $\alpha$-CD-PEG $\$ 20000$ gel is shown in Figure 8 . It is very interesting that the gel-melting temperature showed slow decrease with increase in PEG concentration.

\section{DISCUSSION}

Aqueous solutions of $\alpha-C D$ and PEG of molecular weight higher than 2000 form gels rather than stoichiometric complexes in a crystalline state. The gelation is thought to result from complex formation between partial units of PEG chains and $\alpha-C D$, because gelation does not occur with lack of PEG or $\alpha-C D$ (Figure 2). A better understanding of this idea will be taken with the results of Figures 3 and 4 , that the time of gelation decreases as the concentrations of PEG or $\alpha-\mathrm{CD}$ increase. It is interesting that the time of gelation increases as the molecular weight of PEG increases (Figure 5). This can be explained in terms of the concentration of the end groups of PEG. The PEG chains penetrate the $\alpha$-CD cavities from the two ends of the chains. The concentration of the end groups of PEG decreases with increase in the PEG molecular weight. Therefore the time of gelation increases with decrease in the concentration of PEG end groups.

Thermoreversible gelation in general results from a particular type of crystallization where micro-crystals act as physical cross-links to give rise to a network. ${ }^{7}$ In our system, we think, gel-forming crystallization is not from direct interaction between polymer chains but between $\alpha$-CD-PEG inclusion complex chains, since $\alpha$-CD-PEG complexes show strong crystallinity which makes the complexes separate out from aqueous solutions in cases of PEG molecular weights less than 2000.

One may ask how $\alpha$-CD-PEG complexes are formed and act as cross-linking domains during the gelation of $\alpha$-CD-PEG aqueous solutions for PEG molecular weight higher than 2000. The gels in Figure $6 a-h$ were formed from aqueous solutions in which the molar ratio of ethylene glycol units to $\alpha$-CD was $2: 1$. From the results of Figure 6 there are both complexed $\alpha$-CD and uncomplexed $\alpha$-CD in the gels, and the ratio of complexed $\alpha-C D$ to uncomplexed $\alpha-C D$ in the gels decreases with increase in PEG molecular weight. This indicates that the PEG chain is partially included (covered) by the complexed $\alpha$-CD. (If stoichiometric $\alpha$-CD PEG complexes form in $100 \%$, all $\alpha$-CD would be consumed and no uncomplexed $\alpha-C D$ would remain, because we made the molar ratio $2: 1$ between the ethylene glycol units of PEG and a-CD.) The PEG chains penetrate $\alpha-C D$ cavities from each end so that the partially included units of PEG chains are close to both ends of the polymer chains. Since the number of polymer chains decreases as the molecular weight of PEG increases, gels formed from the higher molecular weight PEG consist of a small fraction of an $\alpha$-CD-PEG complex. (Even though PEG of higher molecular weight may form longer complex domains.) Therefore, the ratio of complexed $\alpha-C D$ to uncomplexed $\alpha-C D$ in gels decreases with increase in PEG molecular weight.

From these results, we suggest one of the most probable configurations for $\alpha$-CD-PEG gels in Figure 9, with the included units of PEG chain by $\alpha-C D$, which result in cross-linkage, and non-included units of PEG chain for each single PEG chain. Therefore, a mechanism for gelation is proposed which can account for the formation of $\alpha$-CD-PEG gels. The PEG chains penetrate $\alpha-C D$ cavities continuously from 


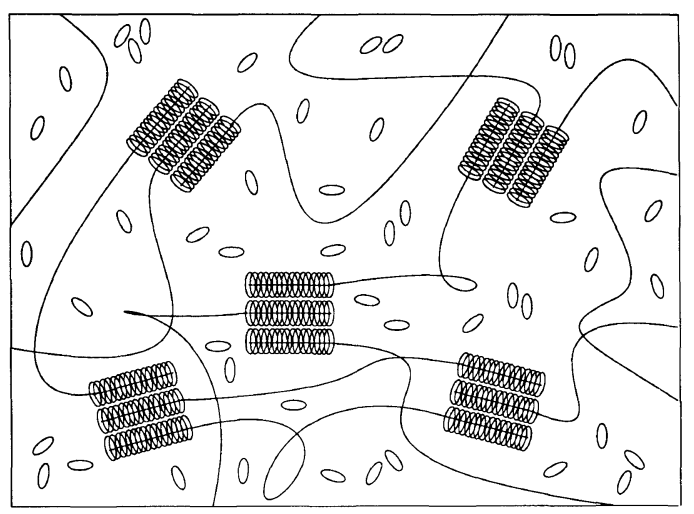

Figure 9. Proposed structure of $\alpha$-CD-PEG gel. represents the $\alpha$-CD-PEG inclusion complex domains which aggregate to micro-crystals which act as physical cross-links giving rise to a network. $\alpha$-CD-PEG complex domains for each PEG chain are near the two ends of PEG chain. This does not mean that the number of $\alpha$-CD-PEG complex domains in a aggregate is limited to three. 0 represents uncomplexed $\alpha$-CD with random alignment in the gel. $\sim$ represents the units of PEG chain, which are not included by $\alpha-\mathrm{CD}$.

each end of the polymer regardless of molecular weight in aqueous solutions. The rings of $\alpha$-CD threaded on the PEG chains connect together by hydrogen bonds between $\mathrm{CDs}$ to form stoichiometric $\alpha$-CD-PEG inclusion complexes partially. The $\alpha$-CD molecules become hydrophobic because of the consumption of hydroxyl groups and $\alpha$-CD-PEG complex domains form aggregates by hydrophobic interaction. For a PEG molecular weight of less than 2000, aggregation takes place after or during each single PEG chain is included by $\alpha-C D$. But for a PEG molecular weight of higher than 2000, aggregation takes place before each PEG chain is wholly included by $\alpha-C D$, i.e., when a PEG chain penetrates $\alpha$-CD cavities from the two ends, the polymer chain is so long that the middle of the chain is not yet included by $\alpha$-CD but inclusion complex domains are long enough to aggregate with other complex domains. These aggregations result in microcrystals which play a role in physical cross-links to give rise to a network. Therefore, the aqueous solutions of high molecular weight
PEG and $\alpha$-CD form gels.

The increase in gel-melting temperature with increase in PEG molecular weight or $\alpha$-CD concentration may be explained by the formation of longer $\alpha$-CD-PEG complex domains which act as stronger cross-links for the gels. In contrast, gel-melting temperature decreases with increase in PEG concentration. It can be thought that the decrease in molar ratio of $\alpha$-CD to each PEG chain due to the increase in the number of PEG chains results in forming shorter $\alpha$-CD-PEG complex domains which act as weaker cross-links for the gels.

\section{CONCLUSIONS}

High molecular weight PEGs were found to form gels with $\alpha-C D$ in aqueous solutions under suitable conditions. Gelation results from formation of inclusion complexes between $\alpha-\mathrm{CD}$ and partial PEG chain units close to both ends. The inclusion complexes of $\alpha-\mathrm{CD}$ with PEG chain units play a role in physical cross-links to give rise to a network. The time of gelation decreases with increase in the concentration of $\alpha$-CD or PEG, but increases with increase in the PEG molecular weight due to decrease in the concentration of the end groups of PEG. Gel-melting temperature measurements showed that the gel-melting temperature increases with increase in the PEG molecular weight or $\alpha$-CD concentration, and decrease with increase in the PEG concentration due to the formation of the longer and shorter $\alpha$-CD-PEG complex domains, respectively.

Acknowledgment. We thank Dr. T. Yamamoto for making measurements of X-ray powder diffraction.

\section{REFERENCES}

1. M. L. Bender and M. Komiyama, "Cyclodextrin Chemistry," Springer-Verlag, New York, 1978. 
2. W. Saenger, Angew. Chem., Int. Ed. Engl., 19, 334 (1980).

3. (a) R. Breslow, Science (Washington, D. C.), 218, 532 (1982). (b) I. Tabushi, Acc. Chem. Res., 15, 66 (1982).

4. (a) Y. Kotake and E. G. Janzen, J. Am. Chem. Soc., 111, 2066 (1989). (b) W.-S. Chung, N. J. Turro, J. Silver, and W. J. Nobel, J. Am. Chem. Soc., 112, 1202 (1990).

5. (a) A. Harada and M. Kamichi, Macromolecules, 23, 2821 (1990). (b) A. Harada, J. Li, and M. Kamachi, Nature, 356, 325 (1992). (c) A. Harada, J. Li, and
M. Kamachi, Macromolecules, 26, 5689 (1993). (d) A. Harada, J. Li, T. Nakamitsu, and M. Kamachi, J. Org. Chem., 58, 7524 (1993).

6. H.-M. Tan, B. H. Chang, E. Bear, and A. Hiltner, Eur. Polym. J., 19, 1012 (1983). (b) M. Komatsu, T. Inoue, and K. Miyasaka, J. Polym. Sci., Polym. Phys. Ed., 24, 303 (1986).

7. (a) M. Girolamo, A. Keller, K. Miyasaka, and N. Overbergh, J. Polym. Sci., Polym. Phys. Ed., 14, 39 (1976). (b) A. Keller, Polymer, 20, 2371 (1979). 